\title{
X-Ray Photoelectron Spectroscopy and Raman Spectroscopy Studies on Thin Carbon Nitride Films Deposited by Reactive RF Magnetron Sputtering
}

\author{
Masao Matsuoka ${ }^{1 *}$, Sadao Isotani ${ }^{1}$, Ronaldo D. Mansano ${ }^{2}$, Wilmer Sucasaire ${ }^{1}$, \\ Ricardo A. C. Pinto ${ }^{1}$, Juan C. R. Mittani ${ }^{1}$, Kiyoshi Ogata ${ }^{3}$, Naoto Kuratani ${ }^{4}$ \\ ${ }^{1}$ Institute of Physics, University of São Paulo, São Paulo, Brazil \\ ${ }^{2}$ Polytechnical School, University of São Paulo, São Paulo, Brazil \\ ${ }^{3}$ Nissin Electric Company, Ltd., Kyoto, Japan \\ ${ }^{4}$ MEMS Development Department, Semiconductor Division, OMRON Corporation, Shiga, Japan \\ Email: *matsuoka@if.usp.br
}

Received March 23, 2012; revised April 22, 2012; accepted May 12, 2012

\begin{abstract}
Thin carbon nitride $\left(\mathrm{CN}_{x}\right)$ films were synthesized on silicon substrates by reactive RF magnetron sputtering of a graphite target in mixed $\mathrm{N}_{2} / \mathrm{Ar}$ discharges and the $\mathrm{N}_{2}$ gas fraction in the discharge gas, $F_{\mathrm{N}}$, varied from 0.5 to 1.0 . The atomic bonding configuration and chemical composition in the $\mathrm{CN}_{x}$ films were examined using X-ray photoelectron spectroscopy (XPS) and the degree of structural disorder was studied using Raman spectroscopy. An increase in the nitrogen content in the film from 19 to 26 at $\%$ was observed at $F_{\mathrm{N}}=0.8$ and found to influence the film properties; normality tests suggested that the data obtained at $F_{\mathrm{N}}=0.8$ are not experimental errors. The interpretation of XPS spectra might not be always straightforward and hence the detailed and quantitative comparison of the XPS data with the information acquired by Raman spectroscopy enabled us to interpret the decomposed peaks in the N 1s and C 1s XPS spectra. Two $\mathrm{N} 1$ 1s XPS peaks at 398.3 and $399.8 \mathrm{eV}$ (peaks $N_{1}$ and $N_{2}$, respectively) were assigned to a sum of pyridine-like nitrogen and $-\mathrm{C} \equiv \mathrm{N}$ bond, and to a sum of pyrrole-like nitrogen and threefold nitrogen, respectively. Further, the peaks $N_{1}$ and $N_{2}$ were found to correlate with C 1s XPS peaks at 288.2 and $286.3 \mathrm{eV}$, respectively; the peak at $288.2 \mathrm{eV}$ might include a contribution of $s p^{3}$ carbon.
\end{abstract}

Keywords: Carbon Nitride; Magnetron Sputtering; X-Ray Photoelectron; Raman Scattering

\section{Introduction}

The prediction of hypothetical material $\beta-\mathrm{C}_{3} \mathrm{~N}_{4}$, whose hardness might be equal or superior to that of diamond [1], has motivated much research to synthesize and characterize carbon-nitrogen materials, because this superhard material has not only scientific interest, but also promising technological potential for thin-film application. Extensive experimental effort has been made on growing thin carbon nitride $\left(\mathrm{CN}_{x}, 0<x<1.33\right)$ films with various deposition methods, such as reactive sputtering [2-4], dual ion beam sputtering [5], ion beam deposition [6-8], ion beam nitridation [9], laser ablation [10], chemical vapor deposition [11].

Despite much effort to achieve the stoichiometric compound, the large majority of the experiments done to date indicate that the maximal achievable nitrogen content structurally incorporated into amorphous $\mathrm{CN}_{x}$ formed is limited to about 30 to 40 at $\%$ less than the stoichiometric

\footnotetext{
${ }^{*}$ Corresponding author.
}

value of the $\beta-\mathrm{C}_{3} \mathrm{~N}_{4}$ phase (57 at $\%$ ) [12]. Some authors reported the presence of nanometer-sized $\beta-\mathrm{C}_{3} \mathrm{~N}_{4}$ crystallites buried in an amorphous $\mathrm{CN}_{x}$ matrix on the basis of electron diffraction data [13], but others claimed that there is no definite evidence of the existence of crystalline $\beta-\mathrm{C}_{3} \mathrm{~N}_{4}$ [14]. Despite the discrepancy in stoichiometry, the obtained $\mathrm{CN}_{x}$ films prove to be interesting and useful in material science and coating technology, because of their high hardness, low friction, wear resistance, and ease of fabrication.

Numerous studies have been carried out on the different kinds of amorphous $\mathrm{CN}_{x}$; however, many questions, fundamentally regarding the identification of carbon-nitrogen bonding configurations, still remain. Carbon as well as nitrogen has some atomic bonding configurations and the most commonly encountered configurations of carbon and nitrogen are linear, trigonal, and tetrahedral, which correspond to the $s p, s p^{2}$, and $s p^{3}$ hybridizations, respectively. Indeed, the carbon coordination in nitrogenfree carbon networks defines the local structural properties 
in which hard solid materials are usually closely related to three-dimensional four $s p^{3}$ orbitals which make strong $\sigma$ bonds with adjacent atoms, while two-dimensional $s p^{2}$ carbon forming three $\sigma$ bonds and one $\pi$ bond will lead to much softer and somewhat brittle materials, due to its two-dimensional structure of hexagonal carbon layers. The addition of nitrogen atoms to the carbon network is capable to modify the carbon coordination in different manners and the $\mathrm{CN}_{x}$ films can hence present a wide variety of bonding configurations [15,16]. Consequently, the optimization of the amorphous $\mathrm{CN}_{x}$ properties requires better assignment of the different types of chemical bonding in the films.

X-ray photoelectron spectroscopy (XPS) technique provides the information on chemical bonds and atomic bonding, based on the chemical shifts in their core levels in a certain chemical environment, and on the surface chemical composition of the material [17-19]. This technique has been used extensively to study $\mathrm{CN}_{x}$ films and there are a great number of publications on XPS spectra of $\mathrm{CN}_{x}$ films. In the case of $\mathrm{CN}_{x}$ films, however, XPS studies of carbon and nitrogen suffer from the lack of spectral resolution; the spectra have broad overlapping peaks, due to the amorphous nature and resulting small chemical shifts, leading sometimes to difficulties in identification and controversial assignments of different chemical environments of carbon and nitrogen [20]. The most difficult task is the bonding identification. On the other hand, Raman spectroscopy is a standard and nondestructive technique to detect the difference in energy between incident and inelastically scattered photons which are associated with different vibration modes and is widely used for the characterization of all kinds of carbon-based materials. Different from XPS, Raman spectroscopy can show both local and collective bond vibration modes and therefore can often supply confused results in case of amorphous carbon. Considerable confusion exists in the interpretation of the spectra obtained by XPS and Raman for $\mathrm{CN}_{x}$ materials. Therefore, it is obvious that complementary additional techniques are important in obtaining a more comprehensive understanding of $\mathrm{CN}_{x}$ films. To our knowledge, such an XPS study of $\mathrm{CN}_{x}$ films in combination with Raman spectroscopy has not been accomplished.

In this study we have prepared thin $\mathrm{CN}_{x}$ films on $\mathrm{Si}(100)$ substrates, using reactive RF magnetron sputtering in mixed $\mathrm{N}_{2} / \mathrm{Ar}$ discharges from a graphite target. The $\mathrm{N}_{2}$ gas fraction in the discharge gas, as a deposition parameter, varied from 0.5 to 1.0 . We have analyzed these deposited films in terms of the atomic bonding structure and chemical composition using XPS. The purpose of this paper is to combine the analysis results of XPS with those of Raman spectroscopy in order to obtain the consistent conclusions on the bonding structure of carbon and nitrogen in the $\mathrm{CN}_{x}$ films.

\section{Experimental}

The $\mathrm{CN}_{x}$ films were deposited on (100)-oriented Si substrates of $75 \mathrm{~mm}$ in diameter by reactive RF magnetron sputtering at $13.56 \mathrm{MHz}$. The target, a high-purity $99.9999 \%$ graphite disc of $15 \mathrm{~cm}$ in diameter and $6 \mathrm{~mm}$ in thickness, was mounted on a planar magnetron electrode at a distance of $10 \mathrm{~cm}$ from a substrate holder. Each substrate was cleaned with Piranha etching $\left(4 \mathrm{H}_{2} \mathrm{SO}_{4}+\right.$ $\left.1 \mathrm{H}_{2} \mathrm{O}_{2}\right)$ and dipped in dilute $\mathrm{HF}$ solution $\left(1: 20 \mathrm{H}_{2} \mathrm{O}\right)$ to remove silicon oxide, followed by a rinse with deionized water, and immediately loaded to the substrate holder.

The deposition chamber was evacuated with a turbomolecular pump to the base pressure of $3 \times 10^{-4} \mathrm{~Pa}$. $\mathrm{N}_{2}$ gas mixed with Ar gas (both $99.9999 \%$ purity) was admitted into the deposition chamber through the respective mass flow controllers, and the $\mathrm{N}_{2}$ gas fraction, $F_{\mathrm{N}}$, defined the $\mathrm{N}_{2}$ gas flow relative to the total gas flow, varied from 0.5 to 1.0 at intervals of 0.1 . Each run of deposition was done with the discharge power of $350 \mathrm{~W}$ at the graphite target, keeping the working pressure at $0.4 \mathrm{~Pa}$ for any chosen $F_{\mathrm{N}}$. The substrate was maintained during the deposition at $90^{\circ} \mathrm{C}$ monitored by a thermocouple which was attached to the substrate holder and the deposition time was 5 min for all the films.

Conventional X-ray photoemission data of the films were collected ex situ from the surface freshly cleaned after a sputter using $2 \mathrm{keV} \mathrm{Ar}{ }^{+}$ion beam for 3 min using a Shimadzu ESCA 750 spectrometer with incident $\mathrm{Mg}$ $\mathrm{K}_{\alpha}$ radiation $(1253.6 \mathrm{eV})$ and the binding energies were calibrated with respect to the $\mathrm{Au} 4 \mathrm{f}_{7 / 2}$ peak at $83.8 \mathrm{eV}$, originated from gold coverage, as external standard, deposited on the surface of each sample. Unpolarized Raman spectra were measured ex situ at room temperature using a Renishaw Raman 2000 spectrometer operating with an $\mathrm{Ar}^{+}$laser excitation line of $514.5 \mathrm{~nm}$. The deposited film thickness, which was measured with a Dektak 3030 surface profilometer, ranged from 97 to $157 \mathrm{~nm}$.

Our study includes fitting of C 1s and N 1s XPS spectra with the Doniach-Šunjić function which has been widely used for peak fitting [21], using the fitting procedure described elsewhere [22]. The fit parameters were optimized with a grid method [23] and the deviations due to the fitting procedure were obtained as follows. Varying each parameter to higher and lower values than its bestfit parameter value, the corresponding cost functions were calculated. The largest deviation of such a parameter value, which gave an increase of about $20 \%$ in the cost function, from the best-fit parameter value, was used as the fitting deviation of the parameter [24].

\section{Results and Discussion}

\subsection{Deposition Rate}

The deposition rate of the films, evaluated by dividing 
the deposited film thickness by the deposition time, is shown in Figure 1 as a function of $\mathrm{N}_{2}$ gas fraction in the discharge gas, $F_{\mathrm{N}}$. As $F_{\mathrm{N}}$ is changed from 0.5 to 1.0 , the deposition rate increases from 0.32 to $0.52 \mathrm{~nm} / \mathrm{s}$. This $F_{\mathrm{N}}$ has a significant influence on the deposition rate of the films and such an increase is observed already in the formation of $\mathrm{CN}_{x}$ films prepared by magnetron sputtering [25].

When $\mathrm{N}_{2}$ gas is mixed into the sputtering atmosphere, it causes a variety of effects. Incident ions generally accumulate on the target and growing film surfaces and can form volatile compounds with surface layer atoms, such as $\mathrm{CN}^{+}, \mathrm{HCN}^{+}, \mathrm{C}_{2} \mathrm{~N}_{2}^{+}$, during the deposition process [26]. The formation of these compounds lowers the surface binding energy and they can be desorbed from the sur- face (commonly referred to as "chemical sputtering"). At conditions in which the ion energy $(<100 \mathrm{eV})$ is below the physical sputtering threshold, the higher the $\mathrm{N}_{2}$ gas fraction in the discharge gas, the faster the formation rate of volatile compounds, and thereby the film formation ceases due to chemical sputtering. For our experimental result the contrary is the case.

Furthermore, during the deposition of amorphous carbon, it is known that, when the energy of an incident ion exceeds certain threshold energy, the ion can penetrate the surface of the growing film and enter an interstitial site, increasing the local density. This process is termed "subplantation" (low energy surface implantation) and the local bonding around this site will reform according to the densification and thermal spike [27].

In any event, we expect that the material removal from the surface of growing film due to chemical sputtering may be offset by a higher deposition flux of film-forming species at higher $\mathrm{N}_{2}$ gas fractions [26]; however, detailed interpretation of the deposition rate is difficult due to the complex subplantation and chemical sputtering processes in the mixed gas system and more experimental research is required as a result.

\subsection{XPS Spectra}

Three elemental species, carbon, nitrogen, and oxygen,

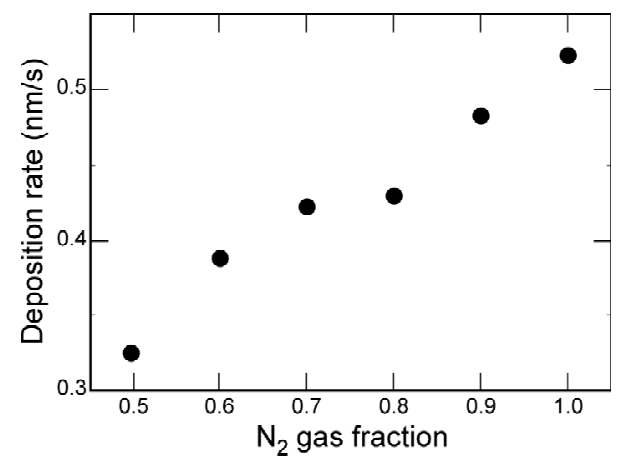

Figure 1. Deposition rate of $\mathbf{C N}_{x}$ films as a function of $\mathbf{N}_{2}$ gas fraction. were identified in the films by assignment of the corresponding signals observed in the XPS spectra. Figures 2(a) and (b) depict the respective $\mathrm{N} 1 \mathrm{~s}$ and $\mathrm{C} 1 \mathrm{~s}$ spectra (solid lines) observed for the film prepared at $F_{\mathrm{N}}=0.7$ along with the individual decomposed peaks which will be explained later. Both the $\mathrm{N} 1 \mathrm{~s}$ and $\mathrm{C} 1 \mathrm{~s}$ spectra, normalized to the same maximum intensity, present asymmetric broadening which implies the presence of some individual peaks related to different atomic bonding configurations. Diverse and, in certain case, contradictory interpretations have been published on the XPS analysis results of $\mathrm{CN}_{x}$ films, due to the presence of complex local environment and the lack of appropriate standard samples for reference.

The nitrogen content in each film can be estimated from $A(\mathrm{~N}) / A_{\text {sum }}$ and $A_{\text {sum }}=[A(\mathrm{~N})+A(\mathrm{C})+A(\mathrm{O})]$, where $A(\mathrm{~N}), A(\mathrm{C})$, and $A(\mathrm{O})$ are the areas under the whole $\mathrm{N} 1 \mathrm{~s}$, $\mathrm{C} 1 \mathrm{~s}$, and $\mathrm{O} 1 \mathrm{~s}$ spectra, respectively, after correcting with relative sensitive factors due to the analyzer transmission and the photoionization cross section.

Table 1 indicates the experimental data of $A(\mathrm{~N}), A(\mathrm{C})$, $A(\mathrm{O}), A_{\text {sum }}, A(\mathrm{~N}) / A_{\text {sum }}, A(\mathrm{C}) / A_{\text {sum }}$, and $A(\mathrm{O}) / A_{\text {sum. }}$. Figure 3 shows the nitrogen content in the film as a function of $F_{\mathrm{N}}$, indicating that: all the films are deficient in nitrogen, deviating considerably from stoichiometric $\mathrm{C}_{3} \mathrm{~N}_{4}(57$ at $\%$ of nitrogen); the nitrogen content is fairly constant at around 19 at $\%$ independent of $F_{\mathrm{N}}$, except for a rise in the nitrogen content to about 26 at $\%$ at $F_{\mathrm{N}}=0.8$.

The nitrogen content may be determined by the competition between the deposition process of nitrogen-containing species by subplantation and the desorption process of volatile species from the surface of growing film by chemical sputtering [28]. This chemical sputtering is selective, depending on bonding and stability of sites where volatile species are incorporated, and the interpretation

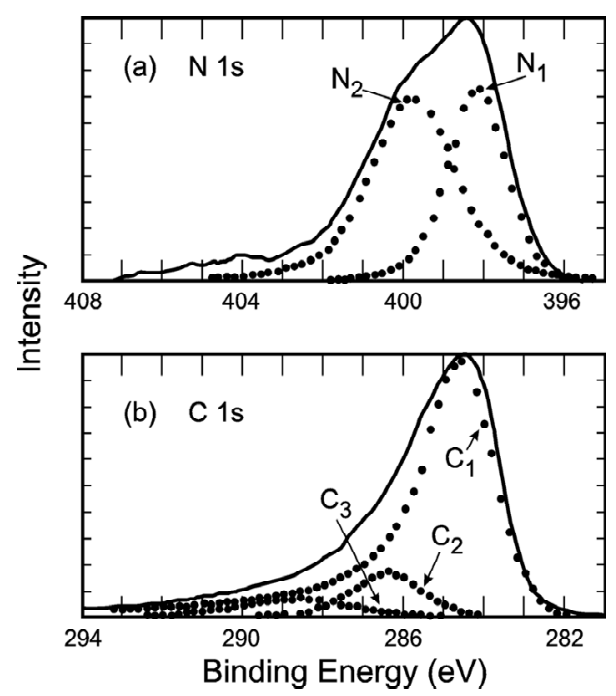

Figure 2. (a) N 1s XPS spectrum and (b) C 1s XPS spectrum of the film deposited at $F_{N}=0.7$. 
Table 1. Areas of N 1 s, C 1 s, and $\mathrm{O} 1 \mathrm{~s}$ XPS spectra. The units of the areas are in counts.eV.

\begin{tabular}{cccccccc}
\hline$F_{\mathrm{N}}$ & $A(\mathrm{~N})$ & $A(\mathrm{C})$ & $A(\mathrm{O})$ & $A_{\text {sum }}$ & $A(\mathrm{~N}) / A_{\text {sum }}$ & $A(\mathrm{C}) / A_{\text {sum }}$ & $A(\mathrm{O}) / A_{\text {sum }}$ \\
\hline 0.5 & 17,175 & 66,981 & 1782 & 85,938 & 0.200 & 0.779 & 0.0207 \\
0.6 & 15,636 & 68,362 & 1321 & 85,319 & 0.183 & 0.801 & 0.0155 \\
0.7 & 15,223 & 61,902 & 2182 & 79,307 & 0.192 & 0.781 & 0.0275 \\
0.8 & 20,545 & 55,708 & 4170 & 80,423 & 0.225 & 0.693 & 0.0519 \\
0.9 & 17,757 & 67,005 & 1733 & 86,495 & 0.205 & 0.775 & 0.0200 \\
1.0 & 14,072 & 62,229 & 2351 & 78,652 & 0.179 & 0.791 & 0.0299 \\
Mean & 16,000 & 65,300 & 1900 & 82,700 & & & \\
$\sigma$ & 3100 & 6300 & 1100 & 5500 & & & 0.012 \\
$p$ value & 0.67 & 0.25 & 0.09 & 0.12 & 0.088 & & 0.13 \\
\hline
\end{tabular}

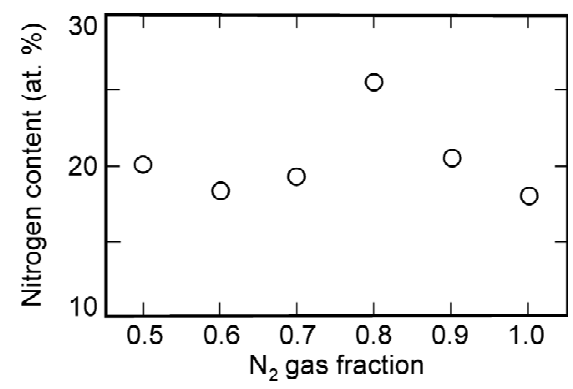

Figure 3. Nitrogen content in the film as a function of $\mathrm{N}_{2}$ gas fraction.

of this result needs further research. Further, from the table, the oxygen content given by $A(\mathrm{O}) / A_{\text {sum }}$ is found to be relatively low ( $2-5$ at $\%)$.

The point corresponding to $F_{\mathrm{N}}=0.8$ in Figure 3 is found to be removed in value from the others. Fearing that this outlying point might be the result of an error in measurement, the data set of $A_{\text {sum }}$ is examined carefully before any further analysis. It is observed at first that: the standard deviation of $A_{\text {sum }}(3600)$ is small compared with the mean (4\%); the difference between the mean and median (82871) is also small $(0.2 \%)$; the means calculated from all the subsets, which are formed by subtracting one data from the data set, are very close to the mean of $A_{\text {sum. }}$. The normality test of Anderson-Darling [29] and the following evaluation by the software ACTION [30] for the normality test are used to determine whether the data set is well-modeled by a normal distribution or not and $p$ value in the test is found to be 0.12 .

From the considerations above mentioned and the criterion of $p$ value $\geq 0.05$ for a normal distribution, the data set of $A_{\text {sum }}$ is distributed normally in spite of a small number of the data set member. Consequently, we can conclude that the XPS measurement was run routinely and correctly. Further, all the data of $A_{\text {sum }}$ fall within a 99\% confidence interval and then this interval is considered as the deviation from the mean of each parameter.

Next the data sets of $A(\mathrm{~N}), A(\mathrm{C})$, and $A(\mathrm{O})$, also given in Table 1, are examined and $p$ values obtained are 0.67, 0.25 , and 0.09 , respectively, suggesting normal distribu- tions for the three data sets; however, the data for $F_{\mathrm{N}}=$ 0.8 are outside $99 \%$ confidence intervals for the three data sets. To reduce the effect of random fluctuations in $A(\mathrm{~N})$ and $A(\mathrm{C})$, their fractional contents, i.e., $A(\mathrm{~N}) / A_{\text {sum }}$ and $A(\mathrm{C}) / A_{\text {sum }}$, which are also given in the table, are examined; $A(\mathrm{O}) / A_{\text {sum }}$ is excluded from the examination due to its relatively small contribution. The $p$ value obtained for the data set of $A(\mathrm{~N}) / A_{\text {sum }}$ indicates a normal distribution $(p=0.088)$ and that for the data set of $A(\mathrm{C}) / A_{\text {sum }}$, a non-normal distribution $(p=0.012)$; the data for $F_{\mathrm{N}}=0.8$ are outside $99 \%$ confidence intervals for the two data sets.

Note that the subset of $A(\mathrm{C}) / A_{\text {sum }}$ without the data for $F_{\mathrm{N}}=0$ shows a normal distribution $(p=0.45)$. Figures 4(a) and (b) depict the relations between the experimental data and the expected data from the normal distribution for the data sets of $A(\mathrm{~N}) / A_{\text {sum }}$ and $A(\mathrm{C}) / A_{\text {sum }}$, respectively, as a function of parameter $Z$, which is known as a standard normal random variable and defined by $Z=(X-\mu) / \sigma$, where $X$ is experimental data, $\mu$ is the mean, and $\sigma$ is the standard deviation. It is known that, if the distribution of data is normal, the plotted points should approximately lie on a straight line. All the points except the point for $F_{\mathrm{N}}=0.8$ in each figure arise from a normal distribution; however, the point for $F_{\mathrm{N}}=0.8$ in each figure does not lie on the line determined by the other points and hence the data for $F_{\mathrm{N}}=0.8$ are outliers.

As shown in detail in the following Sub-Heading 3.3, three distinct Raman parameters are evaluated from the spectra analysis and the normality test results for each Raman parameter indicate that: the parameter value related to the film prepared at $F_{\mathrm{N}}=0.8$ is an outlier and the other parameter values originate from a normal distribution in each data set. Taking into consideration that the two measurements with XPS and Raman are independent, we conclude that the data for $F_{\mathrm{N}}=0.8$ are genuine results and may indicate an extreme behavior of the corresponding film.

The Knoop hardness of the films was tentatively measured on different sites of each film using a microhardness tester with a load of $10 \mathrm{gf}$. The film thickness is insufficient for this measurement, because the hardness 


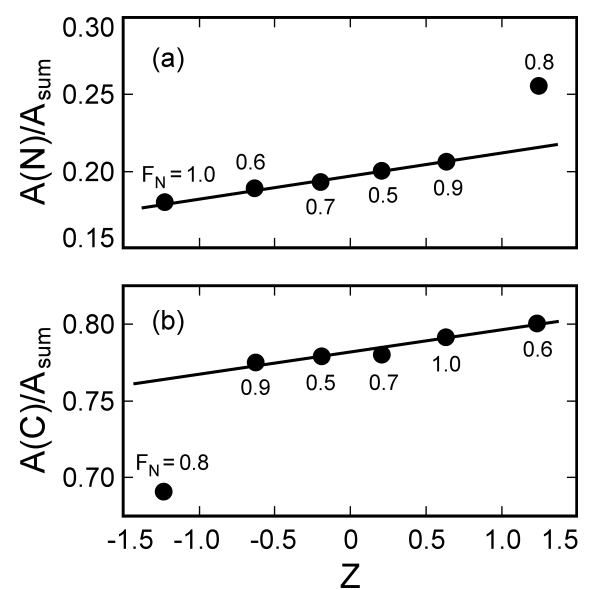

Figure 4. (a) $A(\mathrm{~N}) / A_{\text {sum }}$ and (b) $A(\mathrm{C}) / A_{\text {sum }}$ as a function of $Z$.

reading obtained should be influenced by the silicon substrate with hardness of $10.8 \mathrm{GPa}$. However, it is worth mentioning here that: the apparent hardness reading indicates an abrupt increase from $(5.4 \pm 1.4) \mathrm{GPa}$ to $(13.7 \pm$ $0.8) \mathrm{GPa}$ at $F_{\mathrm{N}}=0.8$; the hardness reading for the film prepared at $F_{\mathrm{N}}=0.8$ is found, through the normality test, to be an outlier. A similar abrupt increase from 18 to 25 $\mathrm{GPa}$ in hardness of $\mathrm{CN}_{x}$ films formed by $\mathrm{RF}$ plasma beam deposition using $\mathrm{N}_{2} / \mathrm{Ar}$ gas mixtures $\left(0.1 \leq F_{\mathrm{N}} \leq 1\right)$ has been found at $F_{\mathrm{N}}=0.5$; however, there is not any explanation for it [31].

The N 1s spectra have been commonly simulated with three or four peaks based on organic molecules containing nitrogen with the well-known atomic bonding configurations. Most authors have used three peaks in the ranges of around $398\left(N_{1}\right), 400\left(N_{2}\right)$, and $402 \mathrm{eV}\left(N_{3}\right)$ $[6,7,9,10]$. The consensus of assignment is that the peak $N_{3}$ is generally attributed to $\mathrm{N}-\mathrm{O}$ bonds and/or chemisorbed $\mathrm{N}_{2}[2,5,6,10]$. Some authors have taken into account an additional peak situated at around $399 \mathrm{eV}$ which is due to $s p$ nitrogen bonded to carbon $(-\mathrm{C} \equiv \mathrm{N})$ forming a nitrile terminating configuration $[5,9]$.

Concerning the peaks $N_{1}$ and $N_{2}$, the majority of authors ascribe the peaks situated below $400 \mathrm{eV}$ to nitrogen bonded to $s p^{3}$ carbon $\left(\mathrm{N}-s p^{3} \mathrm{C}\right)$, and the peaks above 400 $\mathrm{eV}$, to nitrogen linked to $s p^{2}$ carbon $\left(\mathrm{N}-s p^{2} \mathrm{C}\right)$, on the basis of the values of binding energy for urotropine $\left(\mathrm{N}-s p^{3} \mathrm{C}\right)$ at $399.4 \mathrm{eV}$ and pyridine $\left(\mathrm{N}-s p^{2} \mathrm{C}\right)$ at 399.8 $\mathrm{eV}$, respectively $[2,5,6,10]$.

However, this identification scheme is not completely accepted and alternative interpretations are published by some authors. Ronning and co-workers proposed that the peaks $N_{1}$ and $N_{2}$ are due to nitrogen bonded to two and three neighbors, respectively [7]. Muhl and Méndez claimed that the binding energy of nitrogen is governed by the charge transfer of the electron lone pair from the nitrogen according to the surrounding chemical environment; partial or total localization of the lone pair in the $\mathrm{C}-\mathrm{N}$ bond leads to a decrease in the binding energy of the $\mathrm{N} \mathrm{1s} \mathrm{level} \mathrm{and} \mathrm{to} \mathrm{an} \mathrm{increase} \mathrm{in} \mathrm{that} \mathrm{of} \mathrm{the} \mathrm{C} 1 \mathrm{~s}$ level, and less or no localization, to an increase in the binding energy of the $\mathrm{N} 1 \mathrm{~s}$ level and to a decrease in that of the $\mathrm{C} 1 \mathrm{~s}$ level [12]. This charge transfer depends, of course, on the binding nature between carbon and nitrogen. Accordingly, they attributed the peak $N_{1}$ to twofold-coordinated $s p^{2}$ nitrogen in a hexagonal ring as in pyridine (pyridine-like nitrogen) or to nitrogen linked trigonally to three $s p^{3}$ carbon atoms, and the peak $N_{2}$ to threefold-coordinated $s p^{2}$ nitrogen in a pentagonal ring as in pyrrole (pyrrole-like nitrogen). Later Sánchez-Lópes et al. attributed the peak $N_{1}$ to a sum of nitrogen bonded to three $s p^{2}$ carbon atoms $\left(\mathrm{N}-s p^{2} \mathrm{C}\right)$ and terminating bonding configurations $\left(-\mathrm{NH}_{2},-\mathrm{C} \equiv \mathrm{N}\right)$, and the peak $N_{2}$, to pyridine-like nitrogen [3]. On the other hand, Ripalda et al. correlated the peak $N_{1}$ to pyridine-like nitrogen at edges of graphite layers or the overlap of $s p$ and $s p^{2}$ nitrogen, and the peak $N_{2}$ to substitutional nitrogen in graphite, respectively [9]. This assignment is supported by theoretical calculations by Titantah and Lamoen [32]. Further, Normand et al. proposed that the peaks $N_{1}$ and $N_{2}$ may be assigned to $-\mathrm{C} \equiv \mathrm{N}$ bond or pyridine-like nitrogen, and to $s p^{3}$ nitrogen or pyrrole-like nitrogen, respectively, whatever configuration of carbon to which it is bonded [15]. Neidhardt et al. attributed the peak $N_{1}$ to pyridine-like nitrogen and the peak $N_{2}$ to threefold-coordinated nitrogen in graphite layers [4].

The decomposition of the $\mathrm{N}$ 1s spectra in our work was done accordingly in terms of the three peaks $N_{1}-N_{3}$. An example of the results is shown in Figure 2(a); the peaks $N_{1}$ and $N_{2}$, represented by closed circles, are principal. The contribution of the peak $N_{3}$ is not included in the figure, because of the low contamination by oxygen as mentioned above, and hence this peak will be ignored in the following discussion.

The best fitting parameters obtained are given in Table 2 . In this table the asymmetric parameter, $\alpha$, related to the density of states at the Fermi level, and the line width, $\gamma$, related to the lifetime of destruction of the hole states by electron capture are shown; both parameters are used in the Doniach-Šunjić function. No substantial difference is observed in the respective spectra, except for the variation in the integrated intensity of each peak. On the other hand, the interpretation of the $\mathrm{C} 1 \mathrm{~s}$ spectra is even more controversial and not definitive to date. Up to the present, the $\mathrm{C} 1 \mathrm{~s}$ spectra have been generally analyzed by many authors in terms of four peaks around $284.5 \mathrm{eV}\left(C_{1}\right)$ and in the ranges of $285-286\left(C_{2}\right), 287-$ $288\left(C_{3}\right)$, and $288-290 \mathrm{eV}\left(C_{4}\right)$ [5-7]. The consensus is that the peak $C_{1}$ is exclusively attributed to graphite-like or amorphous carbon in the film and adventitious carbon on the surface $[5-7,10]$, and that the peak $C_{4}$ is associated with $\mathrm{C}-\mathrm{O}$ bonds $[5,6]$. 
Table 2. Best-fit parameters for N 1s and C 1s XPS spectra.

\begin{tabular}{ccccc}
\hline & \multicolumn{4}{c}{ XPS parameters } \\
\cline { 2 - 5 } Peak & $\begin{array}{c}\text { Binding } \\
\text { energy (eV) }\end{array}$ & $\alpha$ & $\gamma(\mathrm{eV})$ & $\begin{array}{c}\text { Area } \\
\text { (counts.eV) }\end{array}$ \\
\hline$N_{1}$ & $398.31 \pm 0.15$ & 0.05 & 0.7 & $59 \pm 9$ \\
$N_{2}$ & $399.84 \pm 0.14$ & 0.05 & 1.4 & $83 \pm 10$ \\
$C_{1}$ & $284.70 \pm 0.26$ & 0.26 & 0.8 & $519 \pm 44$ \\
$C_{2}$ & $286.29 \pm 0.30$ & 0.05 & 1.0 & $81 \pm 16$ \\
$C_{3}$ & $288.19 \pm 0.41$ & 0.05 & 1.7 & $52 \pm 8$ \\
\hline
\end{tabular}

Many researchers suggest that the peaks $C_{2}$ and $C_{3}$ are attributed to $s p^{2}$ carbon bounded to nitrogen $\left(s p^{2} \mathrm{C}-\mathrm{N}\right)$ and to $s p^{3}$ carbon linked to nitrogen $\left(s p^{3} \mathrm{C}-\mathrm{N}\right)$, respecttively, referring to the values of binding energy for pyridine $\left(s p^{2} \mathrm{C}-\mathrm{N} ; 285.5 \mathrm{eV}\right)$ and urotropine $\left(s p^{3} \mathrm{C}-\mathrm{N}\right.$; $286.9 \mathrm{eV}$ ) [5]. However, this scheme is not supported by some authors. Ronning et al. postulated that the peak $C_{2}$ and $C_{3}$ are ascribed to $s p^{3}$ carbon bonded to one and two nitrogen atoms, respectively, and expected for the $\beta-\mathrm{C}_{3} \mathrm{~N}_{4}$ structure a single peak at $288 \mathrm{eV}$ which was not observed in their spectra [7]. Sánchez-López et al. required that the peaks $C_{2}$ and $C_{3}$ result from $s p^{2} \mathrm{C}-\mathrm{N}$ inside the aromatic structures and from $s p^{2} \mathrm{C}-\mathrm{N}$ in the aromatic ring attached to an electronegative group $\left(-\mathrm{NR}_{2}\right.$, $-\mathrm{NHR},-\mathrm{C} \equiv \mathrm{N}$, where $\mathrm{R}$ is any other group), respectively [3]. Normand et al. attribute the peak $C_{2}$ to diamond, diamond-like carbon, $s p^{3}$ or $s p^{2}$ carbon singly bonded to nitrogen, and the peak $C_{3}$ to $s p^{3}$ carbon multiply bonded to nitrogen [15].

In our study, the $\mathrm{C} 1 \mathrm{~s}$ spectra observed were fitted accordingly with a combination of the peaks $C_{1}-C_{4}$. Figure 2(b) indicates an example of the decomposition results; the contribution of the peak $C_{4}$ is not included in the figure, due to the low contamination by oxygen. The best fitting parameters obtained are given in Table 2. It is interesting to note that the asymmetric parameter, $\alpha$, leads to conducting properties for the peak $C_{1}$ (graphite or amorphous carbon) and to insulating ones for the other peaks. A change in the $\mathrm{N}_{2}$ gas fraction does not affect the position and width of each decomposed peak, whereas the peak intensity is scaled.

Figure 5(a) shows the relative areas of the decomposed peaks in the $\mathrm{N} 1 \mathrm{~s}$ spectra, i.e., $A\left(N_{1}\right) / A(\mathrm{~N})$ and $A\left(N_{2}\right) / A(\mathrm{~N})$, where $A\left(N_{1}\right)$ and $A\left(N_{2}\right)$ are the corrected areas of the respective peaks $N_{1}$ and $N_{2}$, as a function of $F_{\mathrm{N}}$. Figure 5(b) depicts those in the $\mathrm{C} 1 \mathrm{~s}$ spectra, $A\left(C_{1}\right) / A(\mathrm{C}), A\left(C_{2}\right) / A(\mathrm{C})$, and $A\left(C_{3}\right) / A(\mathrm{C})$, where $A\left(C_{1}\right)$ $A\left(C_{3}\right)$ are the corrected areas of the respective peaks $C_{1}$ $C_{3}$, as a function of $F_{\mathrm{N}}$.

It should be noted from Figure 5 that: 1) there are

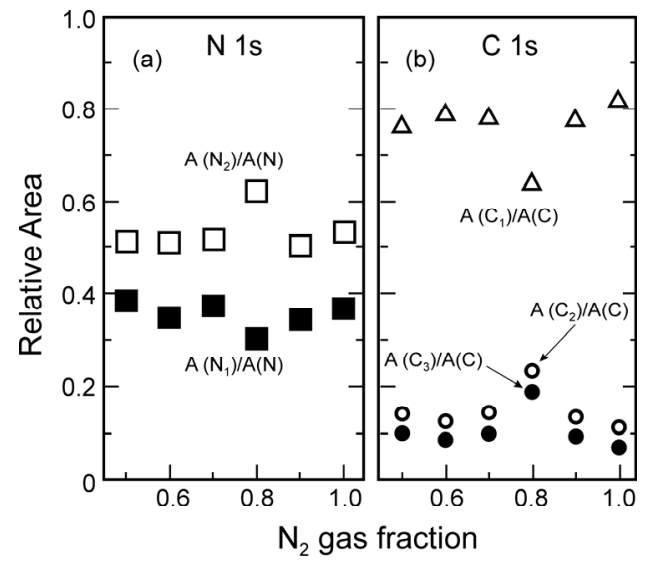

Figure 5. Relative areas of the decomposed peaks in: (a) The $\mathrm{N} 1 \mathrm{~s}$ spectra and (b) The $\mathrm{C} 1 \mathrm{~s}$ spectra, as a function of $\mathrm{N}_{2}$ gas fraction.

distinct changes in all the relative areas at $F_{\mathrm{N}}=0.8$, indicating that a certain change in bonding configurations occurs at this $\mathrm{N}_{2}$ gas fraction; 2) the relative area of the peak $N_{2}$ enhances at the expense of that of the peak $N_{1}$ at $\left.F_{\mathrm{N}}=0.8 ; 3\right)$ the relative area of the peak $C_{1}$ is the major compo- nent in all the $\mathrm{C} 1 \mathrm{~s}$ spectra and the relative areas of the peaks $C_{2}$ and $C_{3}$ grow at the expense of the relative area of the peak $C_{1}$ at $F_{\mathrm{N}}=0.8$. To obtain a certain correlation between one of the N $1 \mathrm{~s}$ XPS peaks and one of the $\mathrm{C} 1 \mathrm{~s}$ XPS peaks, all the combinations are checked and two correlations between $A\left(N_{2}\right)$ and $A\left(C_{2}\right)$ and between $A\left(N_{1}\right)$ and $A\left(C_{3}\right)$ are found, as shown in Figures 6(a) and (b), respectively.

A good correlation between the peaks $N_{2}$ and $C_{2}$ is observed for all the films in Figure 6(a); however, a good correlation between the peaks $N_{1}$ and $C_{3}$ is found for the films except the film formed at $F_{\mathrm{N}}=0.8$ and his exception will be mentioned later. These results mean that: both of the peaks $N_{2}$ and $C_{2}$ are due to the same type of a local chemical environment; both of the peaks $N_{1}$ and $C_{3}$, to the same type of another local chemical environment, except for the film prepared at $F_{\mathrm{N}}=0.8$. It is interesting to notice from Table 2 that the binding energy of the peak $N_{2}$ is higher than that of the peak $N_{1}$, while the binding energy of the peak $C_{2}$ correlating with the peak $N_{2}$ is lower than that of the peak $C_{3}$ correlating with the peak $N_{1}$. These relations may be associated with the charge transfer of the lone pair from the nitrogen in the C-N bond, as mentioned above, that is, the peaks $N_{1}$ and $C_{3}$ can originate from the C-N configuration with the lone pair localized on the nitrogen and the peaks $N_{2}$ and $\mathrm{C}_{2}$ can arise from the C-N configuration with the lone pair delocalized from the nitrogen.

\subsection{Raman Spectra}

Figure 7(a) shows the originally raw Raman spectrum measured in the wave number region of $1000-1800 \mathrm{~cm}^{-1}$ 

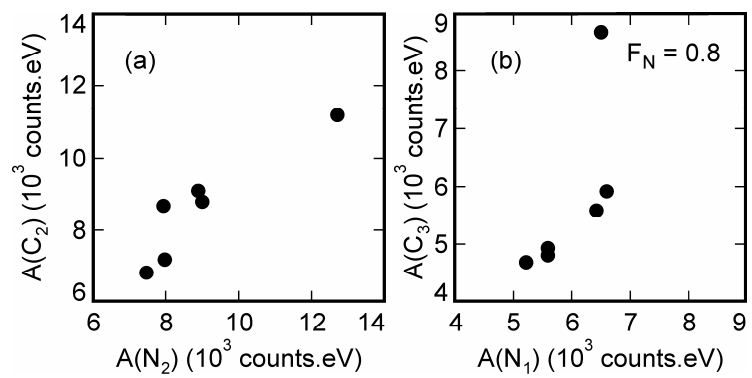

Figure 6. Plots of (a) $A\left(N_{2}\right)$ versus $A\left(C_{2}\right)$ and (b) $A\left(N_{1}\right)$ versus $A\left(C_{3}\right)$.

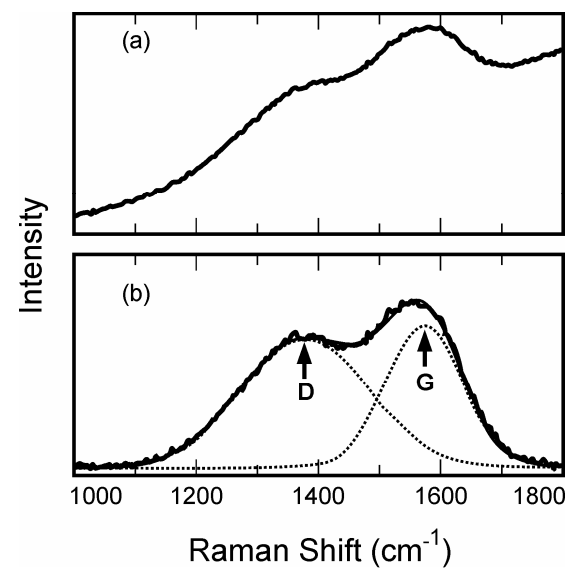

Figure 7. (a) Raw Raman spectrum of the film deposited at $F_{\mathrm{N}}=0.7$; (b) The decomposition results of the spectrum after removal of the background signal.

for the film prepared at $F_{\mathrm{N}}=0.7$; two peaks are observed at around 1350 and $1580 \mathrm{~cm}^{-1}$ in the spectrum. All the Raman spectra obtained in this study indicate these two peaks. Little difference between the Raman spectra of nitrogen-free carbon and those of $\mathrm{CN}_{x}$ is expected in this wave number region, because of the similarity of vibrational frequencies of $\mathrm{C}-\mathrm{C}$ modes to those of $\mathrm{C}-\mathrm{N}$ modes [16]. There is further observed a strong photoluminescence background signal due to recombination of electron hole pairs within $s p^{2}$-bonded clusters [33]. To analyze the two peaks in detail, the parabolic background signal was subtracted from each row spectrum. The subtraction result for the spectrum given in Figure 7(a) is shown in Figure 7(b).

A peak located at $\sim 1350 \mathrm{~cm}^{-1}$ is denominated the $D$ peak and the other at $1550-1590 \mathrm{~cm}^{-1}$, the $G$ peak. The $D$ peak arises from breathing modes $\left(\mathrm{A}_{1 \mathrm{~g}}\right.$ symmetry) of $s p^{2}$ clusters of hexagonal aromatic rings and can correspond to a superposition of ordered and disordered carbon $s p^{3}$ sites, which correspond to crystalline diamond and diamond-like carbon, respectively. On the other hand, the $G$ peak originates from stretching vibrations $\left(\mathrm{E}_{2 \mathrm{~g}}\right.$ symmetry) of any pair of $s p^{2}$ sites in both aromatic rings and olefinic chains and is never observed in the absence of $s p^{2}$ graphite; this peak can provide information on the content and configuration of $s p^{2}$ network $[16,27,34]$. Consequently, most of the carbon-based materials show the Raman spectra dominated by these two peaks.

Figure 7(b) depicts the fitting results, using two Gaussian shapes to the two peaks for the film prepared at $F_{\mathrm{N}}=$ 0.7. According to common practice, three peak parameters: the $G$ peak position, the full width at half maximum (FWHM) of the $G$ peak, and the area ratio of the $D$ to $G$ peak, i.e., $I_{\mathrm{D}} / I_{\mathrm{G}}$, are considered here and these parameters thus determined are shown in Figures 8(a)-(c), respecttively, as a function of $F_{\mathrm{N}}$. Note that all the peak parameters determined exhibit distinct changes at $F_{\mathrm{N}}=0.8$, namely, downward shifts in the $G$ peak position and $I_{\mathrm{D}} / I_{\mathrm{G}}$, and an upward shift in the FWHM. The data sets of $G$ peak position, FWHM, and $I_{\mathrm{D}} / I_{\mathrm{G}}$ are examined using the normality test and $p$ values obtained are $0.15,0.10$, and 0.12 , respectively, suggesting normal distributions for the three data sets; the data for $F_{\mathrm{N}}=0.8$ are found to be always outliers.

In order to understand the behaviors of the peak parameters, we use the three-stage model, which was developed by Ferrari [16] and Robertson [27] to interpret the evolution of the Raman spectra of nitrogen-free amorphous carbon and carbon nitride. This model is applicable to room-temperature deposition and ion implantation of glassy carbon [34]. Starting from perfect crystalline graphite in this model, we consider the dependence of the Raman spectra on clustering of the $s p^{2}$ phase, bond-angle and bond-length disorder, and hybridization, along the

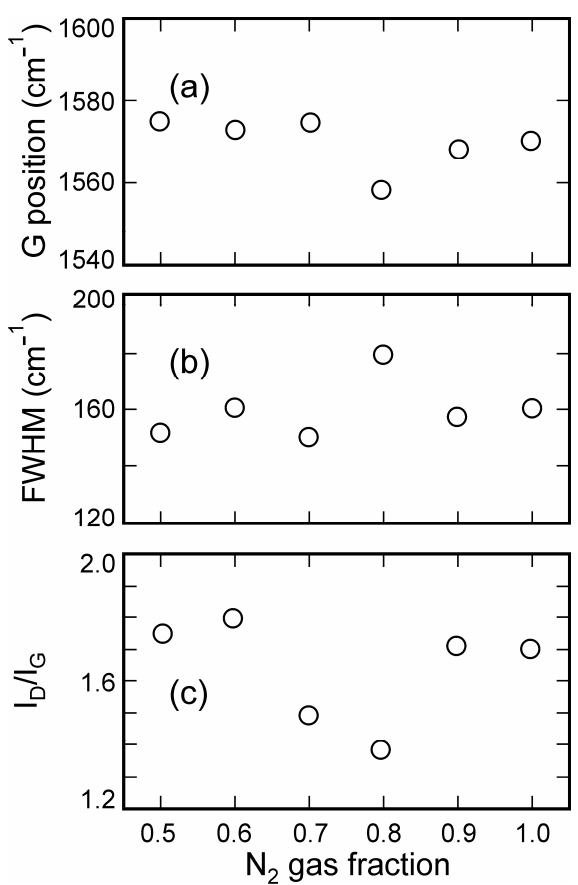

Figure 8. (a) $G$ peak position; (b) FWHM of the $G$ peak; and (c) $I_{\mathrm{D}} / I_{\mathrm{G}}$ ratio, as a function of $\mathrm{N}_{2}$ gas fraction. 
disordering trajectory ranging from ordered graphite to tetrahedral $s p^{3}$ amorphous carbon $(t a-\mathrm{C})$, with three stages. The three stages are as follows: 1) ordered $s p^{2}$ graphite to nanocrystalline ( $n c)$ graphite; 2) $n c$ graphite to $s p^{2}$ amorphous carbon $(a-\mathrm{C})$; (3) $s p^{2} a-\mathrm{C}$ to $t a-\mathrm{C}$ or defected diamond.

The evolution in stage 1 accompanies the progressive clustering of the ordered graphite layers, keeping aromatic rings. This causes the $G$ peak position to shift upward from $1580 \mathrm{~cm}^{-1}$ of ordered graphite to $1600 \mathrm{~cm}^{-1}$ due to phonon confinement and the $D$ mode, which is forbidden in ordered graphite, to appear and enhance. Consequently, $I_{\mathrm{D}} / I_{\mathrm{G}}$ starts increasing from zero according to the Tuinstra-Koenig relation [35]. This relation indicates that: $I_{\mathrm{D}} / I_{\mathrm{G}}$ is inversely proportional to the cluster size of graphite and can reach $I_{\mathrm{D}} / I_{\mathrm{G}} \approx 2$ with the cluster diameter below $2 \mathrm{~nm}[36,37]$. In stage 2, structural disordering of the graphite layers due to the formation of nonsixfold rings and distortion of sixfold rings, and loss of aromatic bonding proceed, but keeping the pure $s p^{2}$ network. This weakens the bonds, lowers the $G$ peak position from 1600 to $1510 \mathrm{~cm}^{-1}$ and reduces the number of the ordered aromatic rings due to the reduction of ordered rings, causing $I_{\mathrm{D}}$ to start decreasing. The $G$ peak retains its intensity and $I_{\mathrm{D}} / I_{\mathrm{G}}$ falls continuously from 2 to $0.1-0.2$ as a result. On passing from stage 2 to stage $3, s p^{2}$ configuration changes gradually from mainly aromatic rings to short olefinic $s p^{2}$ chains, resulting in the absence of the $D$ peak, while the $s p^{3}$ content rises from $\sim 10 \%-20 \%$ to about $85 \%$. Olefinic bonds are shorter than aromatic bonds and have higher vibration frequencies. The $G$ peak position increases from 1510 to $1570 \mathrm{~cm}^{-1}$ and $I_{\mathrm{D}} / I_{\mathrm{G}}$ is very low or zero.

On the basis of this phenomenological three-stage model, all the films deposited in this study can be considered to be in stage 2 , taking into account the $G$ peak position less than that of ordered graphite $\left(1580 \mathrm{~cm}^{-1}\right)$ and $I_{\mathrm{D}} / I_{\mathrm{G}}$ ranging between 1.4 and 1.8 . Thus, the films can be considered to consist of mainly $s p^{2}$ sites and this is consistent with a general remark by Ferrari et al. that $\mathrm{DC}$ or RF magnetron sputtering produces $s p^{2} a-\mathrm{C}: \mathrm{N}$ films [16]. Accordingly, the downward shifts of the $G$ peak position and $I_{\mathrm{D}} / I_{\mathrm{G}}$ at $F_{\mathrm{N}}=0.8$, shown in Figures 8(a) and $8(c)$, respectively, should be attributed to structural disorder in the $s p^{2}$ sites due to further formation of nonsixfold rings, distortion of sixfold rings, and loss of aromatic bonding; the resulting wide variety of $s p^{2}$-bond length and angle distortion leads to the broadening of the $G$ peak, shown in Figure 8(b), as a result [27].

\subsection{Interpretation of the Decomposed Peaks in the $\mathrm{N}$ 1s and $\mathrm{C}$ 1s XPS Spectra}

As mentioned in Sub-Heading 3.2, the currently prevail- ing interpretation of $\mathrm{N} 1 \mathrm{~s}$ and $\mathrm{C} 1 \mathrm{~s}$ XPS spectra, in the base of the values of binding energy for the organic molecules, suggests that the peaks $N_{1}$ and $C_{3}$ are due to $\mathrm{N}-s p^{3} \mathrm{C}$ and $s p^{3} \mathrm{C}-\mathrm{N}$, respectively. Our finding that the structure of all the films produced is amorphous $s p^{2} a-\mathrm{C}$ : $\mathrm{N}$ (Sub-Heading 3.3), and the fact that no evidence of large quantities of $s p^{3}$ carbon is reported in the $\mathrm{CN}_{x}$ films formed by sputtering $[2,4,9,16]$, puts this interpretation in doubt seriously.

It is worth noting that the changes in the relative intensities of the XPS peaks and those in the Raman peak parameters observed at $F_{\mathrm{N}}=0.8$ (Figures 5 and $\mathbf{8}$, respectively) take place at the maximum nitrogen content in the film (26 at $\%$ in Figure 3). Substitutional incorporation of nitrogen into hexagonal graphite layers forms various defects: threefold nitrogen in hexagonal rings, pyridine-like nitrogen, pyrrole-like nitrogen, $-\mathrm{C} \equiv \mathrm{N}$ bond, etc. Pyridine-like twofold nitrogen can be located at an edge of a graphite layer and at a carbon vacancy next to the nitrogen inside a graphite layer [28]. Consequently, pyridine-like nitrogen and $-\mathrm{C} \equiv \mathrm{N}$ bond are terminating defects and their formation contributes to the clustering in the graphite layers [12]. On the other hand, threefold nitrogen and pyrrole-like nitrogen which forms pentagonnal rings in a hexagonal mesh, promote curvature and corrugation in the graphite layers.

The main structural change in stage 1 of the threestage model, as mentioned in Sub-Heading 3.3, is clustering of $s p^{2}$ graphite layers, and that in stage 2 is further structural disordering due to the formation of nonsixfold rings and distortion of sixfold rings. Accordingly, it is expected that the increase in nitrogen content in the film in stage 2 from 19 to 26 at $\%$ at $F_{\mathrm{N}}=0.8$ keeps the clustering of graphite layers intact, while this increase promotes further formation of nonsixfold rings and distortion of sixfold rings. Figure 9 exhibits the fraction of nitrogen content due to the peak $N_{1}$, given by $A\left(N_{1}\right) / A_{\text {sum }}$, and that due to the peak $N_{2}$, given by $A\left(N_{2}\right) / A_{\text {sum }}$, as a function of $F_{\mathrm{N}}$. From the figure the former is almost independent of $F_{\mathrm{N}}$, while the latter shows a prominent rise at $F_{\mathrm{N}}=0.8$. From the above discussion, the experimental finding shown in Figure 9 can be reasonably explained by assuming that: the peak $N_{1}$ is due to terminating defects, such as pyridine-like nitrogen, $-\mathrm{C} \equiv \mathrm{N}$ bond, and the peak $N_{2}$, to pyrrole-like nitrogen and substitutional threefold nitrogen. This identification is in accord with insight obtained from the charge transfer of the lone pair of nitrogen, as mentioned before, and agrees with experimental data obtained for pyridine and pyrrole by XPS (398.6 \pm 0.3 and $400.5 \pm 0.3 \mathrm{eV}$, respectively) [38] and by near-edge X-ray absorption fine structure (NEXAFS) (399.9 and $403.8 \mathrm{eV}$, respectively) [39]. Further, this is consistent with the interpretation published for the individual peaks in the $\mathrm{N} 1 \mathrm{~s}$ XPS spectra $[4,7,9,12,15,32]$. 


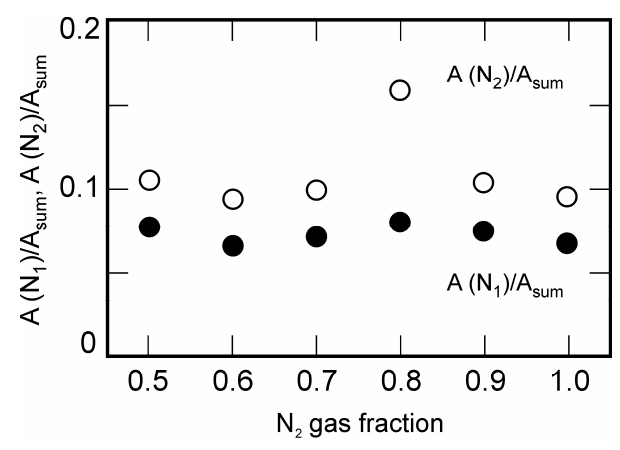

Figure 9. $A\left(N_{1}\right) / A_{\text {sum }}$ and $A\left(N_{2}\right) / A_{\text {sum }}$ as a function of $N_{2}$ gas fraction.

The correlation results, shown in Figure 6, suggest that the peaks $C_{2}$ and $C_{3}$ can be fundamentally assigned to a sum of pyrrole-like nitrogen and substitution threefold nitrogen, and to the terminating defects, respectively; however, as shown in Figure 6(b), the point corresponding to the film formed at $F_{\mathrm{N}}=0.8$ is found to deviate in value from the correlation, indicated by the other films, between the peaks $N_{1}$ and $C_{3}$. This may be explained in the following manner. A theoretical work shows that: a transition from planar to corrugated graphite layers occurs at nitrogen contents above 20 at $\%$; with further increase in nitrogen content to $50 \mathrm{at} \%$, the corrugated clusters are not chemically stable and the three-dimensional $\mathrm{CN}_{x}$ materials emerge [40]. Experimentally, it is reported that $\mathrm{CN}_{x}$ films formed by reactive magnetron sputtering present fullerene-like microstructures, when the nitrogen content reaches $\sim 20$ at $\%[2,41,42]$. This can reasonably explain the increase in hardness reading at $F_{\mathrm{N}}=0.8$, mentioned in Sub-Heading 3.2. Substituting a threefold nitrogen atom for an $s p^{2}$ carbon atom in a graphite layer breaks a $\pi$ bond and leaves an unpaired electron of a remaining carbon atom available to form a $\sigma$ bond to a carbon atom in a similar situation on an adjacent cluster. Thus, the deviation of the film prepared at $F_{\mathrm{N}}=0.8$ from the correlation shown in Figure 6(b) may be attributed to the emergence of $s p^{3}$ carbon, which contributes to an increase in intensity of the peak $C_{3}$. This attribution is consistent with the prevailing identification of the peak $C_{3}\left(s p^{3} \mathrm{C}-\mathrm{N}\right)$.

We admit that the above discussion is rather speculative and that more detailed studies including hardness and transmission electron microscopy measurements would be necessary to get more information. However, we consider that it is worthwhile to remark the detailed and quantitative analysis of the XPS spectra and the consistency in the simultaneous interpretations of Raman and XPS spectra.

\section{Conclusion}

We have studied the atomic bonding configuration and degree of structural disorder in the $\mathrm{CN}_{x}$ films prepared with reactive magnetron RF sputtering, with XPS and Raman spectroscopy, respectively, verifying the obtained data with the normality tests. The Raman results suggest that all the films prepared are amorphous $s p^{2}$ carbon doped with nitrogen in stage 2 of the three-stage model, and also indicate that the increase in the nitrogen content in the film from 19 to 26 at $\%$, keeping the clustering intact, promotes further disorder in the graphite layer. The peak $N_{1}(398.3 \mathrm{eV})$ is little affected in the fraction of nitrogen content by the increase in the nitrogen content in the film, whereas the peak $N_{2}(399.8 \mathrm{eV})$ is enhanced in the fraction of nitrogen content. From the XPS results in combination with the Raman results, it is possible to assign the peak $N_{1}$ to a sum of pyridine-like nitrogen and $-\mathrm{C} \equiv \mathrm{N}$ bond, and the peak $N_{2}$ to a sum of pyrrole-like nitrogen and threefold nitrogen. Further, it has been found from the XPS analysis that: the peak $N_{1}$ correlates with the peak $C_{3}(288.2 \mathrm{eV})$ and the peak $N_{2}$, with the peak $C_{2}(286.3 \mathrm{eV})$; the peak $C_{3}$ may include a contribution of $s p^{3}$ carbon. We could obtain these consistent assignments, when combining the results of XPS with those of Raman spectroscopy; the assignments are in line with the theoretical prediction for the structural evolution induced by nitrogen incorporated into graphite.

\section{Acknowledgements}

This work was supported partially by Japan International Cooperation Agency (JICA), Conselho Nacional de Desenvolviment Científico e Tecnológico ( $\mathrm{CNPq})$, and Coordenação de Aperfeiçoamento de Pessoal de Nível Superior (CAPES).

\section{REFERENCES}

[1] A. Y. Liu and M. L. Cohen, "Prediction of New Low Compressibility Solids," Science, Vol. 245, No. 4920, 1989, pp. 841-842. doi:10.1126/science.245.4920.841

[2] N. Hellgren, M. P. Johansson, E. Broitman, L. Hultman and J.-E. Sundgren, "Role of Nitrogen in the Formation of Hard and Elastic $\mathrm{CN}_{x}$ Thin Films by Reactive Magnetron Sputtering," Physical Review B, Vol. 59, No. 7, 1999, pp. 5162-5169. doi:10.1103/PhysRevB.59.5162

[3] J. C. Sánchez-López, C. Donnet, F. Lefèbvre, C. Fernández-Ramos and A. Fernández, "Bonding Structure in Amorphous Carbon Nitride: A Spectroscopic and Nuclear Magnetic Resonance Study," Journal of Applied Physics, Vol. 90, No. 2, 2001, pp. 675-681. doi:10.1063/1.1380998

[4] J. Neidhardt and L. Hultman, "Beyond $\beta-\mathrm{C}_{3} \mathrm{~N}_{4}$-FullerenLike Carbon Nitride: A Promising Coating Material," Journal of Vacuum Science and Technology A, Vol. 25, No. 4, 2007, pp. 633-644. doi:10.1116/1.2738505

[5] C. Quirós, P. Prieto, A. Fernández, E. Elizalde, C. Morant, R. Schlögl, O. Spillecke and J. M. Sanz, "Bonding and 
Morphology Study of Carbon Nitride Films Obtained by Dual Ion Beam Sputtering," Journal of Vacuum Science and Technology A, Vol. 18, No. 2, 2000, pp. 515-523. doi:10.1116/1.582218

[6] D. Marton, K. J. Boyd, A. H. Al-Bayati, S. S. Todorov and J. W. Rabalais, "Carbon Nitride Deposited Using Energetic Species: A Two-Phase System," Physical Review Letters, Vol. 73, No. 1, 1994, pp. 118-121. doi:10.1103/PhysRevLett.73.118

[7] C. Ronning, H. Feldermann, R. Merk and H. Hofsäss, "Carbon Nitride Deposited Using Energetic Species: A Reviews on XPS Studies," Physical Review B, Vol. 58, No. 4, 1998, pp. 2207-2215.

doi:10.1103/PhysRevB.58.2207

[8] W. Sucasaire, M. Matsuoka, K. C. Lopes, J. C. R. Mittani, L. H. Avanci, J. F. D. Chubaci, N. Added, V. Trava and E. J. Corat, "Raman and Infrared Spectroscopy Studies of Carbon Nitride Films Prepared on Si(100) Substrates by Ion Beam Assisted Deposition," Journal of the Brazilian Chemical Society, Vol. 17, No. 6, 2006, pp. 1163-1169. doi:10.1590/S0103-50532006000600014

[9] J. M. Ripalda, E. Román, N. Díaz, L. Galán, I. Montero, G. Comelli, A. Baraldi, S. Lizzit, A. Goldoni and G. Paolucci, "Correlation of X-Ray Absorption and X-Ray Photoelectron Spectroscopies in Amorphous Carbon Nitride," Physical Review B, Vol. 60, No. 6, 1999, pp. R3705-R3708. doi:10.1103/PhysRevB.60.R3705

[10] R. McCann, S. S. Roy, P. Papakonstantinou, J. A. McLaughlin and S. C. Ray, "Spectroscopic Analysis of $a-\mathrm{C}$ and $a-\mathrm{CN}_{x}$ Films Prepared by Ultrafast High Repetition Rate Pulsed Laser Deposition," Journal of Applied Physics, Vol. 97, No. 7, 2005, pp. 073522-1 - 073522-11. doi:10.1063/1.1874300

[11] Y. Zhang, Z. Zhou and H. Li, "Crystalline Carbon Nitride Films Formation by Chemical Vapor Deposition," $A p$ plied Physics Letters, Vol. 68, No. 5, 1996, pp. 634-636. doi:10.1063/1.116492

[12] S. Muhl and J. M. Méndez, "A Review of the Preparation of Carbon Nitride Films," Diamond and Related Materials, Vol. 8, No. 10, 1999, pp. 1809-1830. doi:10.1016/S0925-9635(99)00142-9

[13] C. Niu, Y. Z. Lu and C. M. Lieber, "Experimental Realization of the Covalent Solid Carbon Nitride," Science, Vol. 261, No. 5119, 1993, pp. 334-337. doi:10.1126/science.261.5119.334

[14] S. Matsumoto, E.-Q. Xie and F. Izumi, "On the Validity of the Formation of Crystalline Carbon Nitride, $\mathrm{C}_{3} \mathrm{~N}_{4}$," Diamond and Related Materials, Vol. 8, No. 7, 1999, pp. 1175-1182. doi:10.1016/S0925-9635(99)00103-X

[15] F. Le Normand, J. Hommet, T. Szörényi, C. Fuchs and E. Fogarassy, "XPS Study of Pulsed Laser Deposited $\mathrm{CN}_{x}$ Films," Physical Review B, Vol. 64, No. 23, 2001, pp. 235416-1-235416-15. doi:10.1103/PhysRevB.64.235416

[16] A. C. Ferrari, S. E. Rodil and J. Robertson, "Interpretation of Infrared and Raman Spectra of Amorphous Carbon Nitride," Physical Review B, Vol. 67, No. 15, 2003, pp. 155306-1-155306-20. doi:10.1103/PhysRevB.67.155306
[17] M. Matsuoka, S. Isotani, J. C. R. Mittani, J. F. D. Chubaci, K. Ogata and N. Kuratani, "Effects of Arrival Rate and Gas Pressure on the Chemical Bonding and Composition in Titanium Nitride Films Prepared on Si(100) Substrates by Ion Beam and Vapor Deposition," Journal of Vacuum Science and Technology A, Vol. 23, No. 1, 2005, pp. 137141. doi:10.1116/1.1839895

[18] M. Matsuoka, S. Isotani, W. Sucasaire, N. Kuratani and K. Ogata, "X-Ray Photoelectron Spectroscopy Analysis of Zirconium Nitride-Like Films Prepared on Si(100) Substrates by Ion Beam Assisted Deposition," Surface and Coating Technology, Vol. 202, No. 13, 2008, pp. 31293135. doi:10.1016/i.surfcoat.2007.11.019

[19] M. Matsuoka, S. Isotani, W. Sucasaire, L. S. Zambom and K. Ogata, "Chemical Bonding and Composition of Silicon Nitride Films Prepared by Inductively Coupled Plasma Chemical Vapor Deposition," Surface and Coating Technology, Vol. 204, No. 18-19, 2010, pp. 29232927. doi.org/10.1016/j.surfcoat.2010.02.071

[20] S. Neuville and A. Matthews, "A Perspective on the Optimization of Hard Carbon and Related Coatings for Engineering Applications," Thin Solid Films, Vol. 515, No. 17, 2007, pp. 6619-6653. doi:10.1016/j.tsf.2007.02.011

[21] S. Doniach and M. Šunjić, "Many-Electron Singularity in X-Ray Photoemission an X-Ray Line Spectra from Metals," Journal of Physics C, Vol. 3, No. 2, 1970, pp. 285291.

[22] M. Matsuoka, S. Isotani, S. Miyake, Y. Setsuhara, K. Ogata and N. Kuratani, "Effects of Ion Energy and Arrival Rate on the Composition of Zirconium Oxide Films Prepared by Ion-Beam Assisted Deposition," Journal of Applied Physics, Vol. 8, No. 2, 1996, pp. 1177-1181. doi:10.1063/1.362855

[23] S. Isotani and A. T. Fujii, "A Grid Procedure Applied to the Determination of Parameters of a Kinetic Process," Computer Physics Communications, Vol. 151, No. 1, 2003, pp. 1-7. doi:10.1016/S0010-4655(02)00692-6

[24] S. Isotani, A. R. Blak and S. Watanabe, "UV Optical Absorption Spectra Analysis of Beryl Crystals from Brazil," Physica B: Condensed Matter, Vol. 405, No. 6, 2010, pp. 1501-1508. doi:10.1016/j.physb.2009.12.029

[25] Y. Kusano, J. E. Evetts, R. E. Somekh and I. M. Hutchings, "Properties of Carbon Nitride Films Deposited by Magnetron Sputtering," Thin Solid Films, Vol. 332, No. 1-2, 1998, pp. 56-61. doi:10.1016/S0040-6090(98)00983-3

[26] J. Neidhardt, L. Hultman, B. Abendroth, R. Gago and W. Möller, "Diagnostics of a $\mathrm{N}_{2} /$ Ar Direct Current Magnetron Discharge for Reactive Sputter Deposition of Fullerene-Like Carbon Nitride Thin Films," Journal of Applied Physics, Vol. 94, No. 11, 2003, pp. 7059-7066. doi:10.1063/1.1625091

[27] J. Robertson, "Diamond-Like Amorphous Carbon," Materials Science and Engineering: R: Reports, Vol. 37, No. 4-6, 2002, pp. 129-281. doi:10.1016/S0927-796X(02)00005-0

[28] L. Hultman, J. Neidhardt, N. Hellgren, H. Sjöström and J.-E. Sundgren, "Fullerene-Like Carbon Nitride: A Resilient Coating Material," MRS Bulletin, Vol. 28, No. 3, 
2003, pp. 194-202. doi:10.1557/mrs2003.62

[29] T. W. Anderson and D. A. Darling, "Asymptotic Theory of Certain Goodness of Fit Criteria Based on Stochastic Processes," Annals of Mathematical Statistics, Vol. 23, No. 2, 1952, pp. 193-212. doi:10.1214/aoms/1177729437

[30] User Manual of the Software Action, "Teste de Anderson-Darling," Chapter 6.3.

http://www.portalaction.com.br/content/63-teste-de-ander son-darling

[31] F.-R. Weber and H. Oechsner, "Properties of Carbon Nitride Layers Generated by Direct Plasma Beam Deposition," Surface and Coatings Technology, Vol. 74-75, Part 2, 1995, pp. 704-709. doi:10.1016/0257-8972(95)08269-7

[32] J. T. Titantah and D. Lamoen, "Carbon and Nitrogen 1s Energy Levels in Amorphous Carbon Nitride Systems: XPS Interpretation Using First-Principles," Diamond and Related Materials, Vol. 16, No. 3, 2007, pp. 581-588. doi:10.1016/i.diamond.2006.11.048

[33] B. Marchon, J. Gui, K. Grannen, G. C. Rahch, J. W. Ager III, S. R. P. Silva and J. Robertson, "Photoluminescence and Raman Spectroscopy in Hydrogenated Carbon Films," IEEE Transactions on Magnetics, Vol. 33, No. 5, 1997, pp. 3148-3150. doi:10.1109/20.617873

[34] A. C. Ferrari and J. Robertson, "Interpretation of Raman Spectra of Disordered and Amorphous Carbon," Physical Review B, Vol. 61, No. 20, 2000, pp. 14095-14107. doi:10.1103/PhysRevB.61.14095

[35] F. Tuinstra and J. L. Koenig, "Raman Spectra of Graphite," The Journal of Chemical Physics, Vol. 53, No. 3, 1970, pp. 1126-1130. doi:10.1063/1.1674108

[36] J. Schwan, S. Ulrich, V. Batori, H. Ehrhardt and S. R. P.
Silva, "Raman Spectroscopy on Amorphous Carbon Films," Journal of Applied Physics, Vol. 80, No. 1, 1996, pp. 440-447. doi:10.1063/1.362745

[37] M. Lejeune, S. Charvet, A. Zeinert and M. Benlahsen, "Optical Behavior of Reactive Sputtered Carbon Nitride Films during Annealing," Journal of Applied Physics, Vol. 103, No. 1, 2008, pp. 0135071-0135078. doi: $10.1063 / 1.2828166$

[38] J. R. Pels, F. Kapteijn, J. A. Moulijn, Q. Zhu and K. M. Thomas, "Evolution of Nitrogen Functionalities in Carbonaceous Materials during Pyrolysis," Carbon, Vol. 33, No. 11, 1995, pp. 1641-1653. doi:10.1016/0008-6223(95)00154-6

[39] Q. Zhu, S. L. Money, A. E. Russell and K. M. Thomas, "Determination of the Fate of Nitrogen Functionalities in Carbonaceous Materials during Pyrolysis and Combustion Using X-Ray Absorption Near Edge Structure Spectroscopy," Langmuir, Vol. 13, No. 7, 1997, pp. 2149-2157. doi:10.1021/la961027s

[40] M. C. Santos and F. Alvarez, "Nitrogen Substitution of Carbon in Graphite: Structure Evolution toward Molecular Forms," Physical Review B, Vol. 58, No. 20, 1998, pp. 13918-13924. doi:10.1103/PhysRevB.58.13918

[41] I. Jiménez, R. Gago, J. M. Albella, D. Cáceres and I. Vergara, "Spectroscopy of Bonding in Hard Graphitic Carbon Nitride Films: Superstructure of Basal Planes and Hardening Mechanisms," Physical Review B, Vol. 62, No. 7, 2000, pp. 4261-4264. doi:10.1103/PhysRevB.62.4261

[42] H. Sjöström, S. Stafström, M. Boman and J.-E. Sundgren, "Superhard and Elastic Carbon Nitride Thin Films Having Fullerenelike Microstructure," Physical Review Letters, Vol. 75, No. 7, 1995, pp. 1336-1339. doi:10.1103/PhysRevLett.75.1336 